\title{
EFFECTS OF CELL PHONE CONVERSATIONS ON DRIVER PERFORMANCE WHILE DRIVING UNDER HIGHWAY MONOTONY
}

\author{
Mark Chan \& Paul Atchley \\ University of Kansas \\ Lawrence, Kansas, USA \\ Email: $\underline{\text { mchan5@,ku.edu }}$
}

\begin{abstract}
Summary: It has often been suggested by individuals that engaging in a cell phone conversation would help keep them awake under monotonous conditions where task underload might lead to a decrease in arousal. To further extend laboratory findings of performance in vigilance type tasks while distracted, a monotonous highway driving scenario was designed to test the anecdotal hypothesis of improved performance. Driver performance related to lane keeping and recall memory were studied under distracted and non-distracted conditions. Results of the simulator study were consistent with laboratory findings of performance decrement when dual tasking indicating that the perceived benefits from the secondary conversational task does not outweigh its costs.
\end{abstract}

\section{INTRODUCTION}

A well observed phenomenon that has been often reported in the vigilance literature is the vigilance decrement (Davies \& Parasuraman, 1982). The decrement is described as a gradual decrease of performance with time on task. It has also been suggested that monotonous highway driving could be likened to a vigilance task (Thiffault \& Bergeron, 2003).

The negative effects of dual tasking have been well documented in simulated driving and simulator tasks. Strayer and Johnson (2001) reported that participants in a tracking task were slower to respond to targets, and were also more susceptible to missing targets when engaged in a cell phone conversation. Atchley and Dressel (2004) reported that participants had a markedly smaller functional field of view when engaged in a cell phone conversation. In a study designed to investigate the potential detrimental effects of speech comprehension and production on driving performance, Kubose et al. (2006) reported that both comprehension and production tasks impaired driver performance with respect to the ability to maintain steady headway distance from a lead car.

Chan (2008) recently suggested that there might be a potential benefit to conversing on a cell phone while engaging in a laboratory vigilance task, but the reported benefit did not sufficiently outweigh its cost. Task performance of participants dual tasking only reached a level similar to participants who had undergone a reduction of vigilance. It has often been suggested by drivers that engaging in cell phone conversations while driving under monotonous and undemanding conditions would help keep them awake. This would be especially true since driving has often been viewed as an automatic task by most experienced drivers. Furthermore, task automaticity would imply a decrease in attentional resources to the task (Schneider \& Shiffrin, 1977), suggesting that participants no longer think about the actual driving task leading to a potential task underload which might affect performance as suggested by Yerkes and Dodson (1908). It has often been suggested by drivers that engaging in a cell phone conversation keeps them awake 
when under-aroused due to the driving task. Drory (1985) reported that a short secondary verbal task of having to periodically report odometer readings did improve driver performance when they were fatigued and underaroused. More recently, Gershon, Ronen, Oron-Gilad and Shinar (2009) reported that an interactive trivia type game had a positive impact on driving performance when drivers began to show signs of under-arousal as induced by a prolonged driving task. In line with what was found by previous work on secondary tasks on drivers who were experiencing task underload, this study sought to investigate the effects of a continuous secondary verbal task, similar to a cell phone conversation, on task performance, and whether or not the benefits of a conversation would outweigh its costs.

\section{METHOD}

\section{Subjects}

Thirty students from the University of Kansas undergraduate research pool, and students from an upper level psychology class, 10 males and 20 females, averaging 21.06 years $(S D=1.41)$, participated for course credit. They had an average of 5.3 years of driving experience $(S D=$ 1.58). Participants were randomly assigned into one of three task conditions: no conversation, 4 males and 6 females; full conversation, 3 males and 7 females; late conversation, 3 males and 7 females. All participants reported English as their primary/native language. A Snellen Visual Acuity Chart was used to test for visual acuity in participants. All participants had normal to corrected visual acuity of 20/32 or better. One participant was disqualified and replaced due to not having a driver's license.

\section{Driving Task}

Participants from all three task types drove in the same simulated environment. The roadway was a four-lane rural highway. Each lane was $12 \mathrm{ft}(3.66 \mathrm{~m})$ in width. A $12 \mathrm{ft}(3.66 \mathrm{~m})$ median separated traffic from the opposite direction. There were occasional curves and hills on the roadway to ensure a degree of realism. There were periods of intermittent traffic from the opposite direction and in the participant's lane. Traffic in the participant's lane travelled at speeds lower than the participant, thus allowing for passing to take place. The total distance of the drive was $140000 \mathrm{ft}(42.67 \mathrm{~km})$. No data was collected in the first $5000 \mathrm{ft}(1.52 \mathrm{~km})$ of the drive to allow participants to reach the specified speed of $65 \mathrm{mph}(104.6 \mathrm{kph})$. As the system did not have a cruise control function participants were instructed to fully depress the accelerator to achieve the maximum speed of $65 \mathrm{mph}(104.6 \mathrm{kph})$. Data collection began from the $5000 \mathrm{ft}(1.52$ $\mathrm{km})$ mark till the $137000 \mathrm{ft}(41.76 \mathrm{~km})$ mark. Data collection was broken into 5 time blocks, each block lasting $26400 \mathrm{ft}(8.05 \mathrm{~km})$.

Wind gusts. To increase the difficulty of the task, a continuous wind gust lasting 30 seconds was introduced within each block except the penultimate block. Participants were thus required to make effortful control of the car to ensure safe driving.

Critical event. To further investigate whether participants were paying attention to the drive, critical events occurred in the first and last block of the drive. In both blocks, a stationary car on the road shoulder would suddenly pull out onto the road when the participant's vehicle was one second away from it (the timing was based on the participant's current speed). 
Memory task. Within each driving block, a billboard image of popular North American fast food restaurants would be presented on the right shoulder. A total of 5 billboards were presented during the drive. This served as a memory task for participants at the end of the driving task. To ensure that participants were not actively trying to remember the billboards, the experimenter did not inform the participant that they were required to remember the billboards.

\section{Conversation Task}

71 words were chosen from the ANEW wordlist (Bradley, \& Lang, 1999) to serve as stimuli for the conversational task. The words were presented randomly via E-prime, and the wordlist was programmed to not repeat any given word until the current wordlist completed its cycle; following which another randomized wordlist would be generated. Participants were told to free associate their responses to the stimulus word. Words were presented one at a time mimicking a back and forth conversational style, with the participant giving a one word response to the word that was presented. If participants failed to respond due to an inability to comprehend the word or the inability to produce a word within the interstimulus-interval (ISI), the software would automatically continue with the next word. The presentation of words was controlled by E-prime with a constant ISI of four seconds. Words were presented to the right ear of the participant via headphones similar to hands-free devices. Participants in the full conversation task group were engaged in the conversation from the beginning of the drive till the end of the scenario.

Participants in the late conversation task only engaged in conversation during the last time block of the scenario.

\section{Performance Metrics}

Analysis of driving performance was done only on the events of the first and last time blocks. The initial goal was to investigate the possible differences in crash avoidance between participants who were conversing on cell phones and those who were not, but analysis did not show any statistical difference between groups in either time blocks. Thus analysis was done on investigating the lane keeping performance of participants on all three task conditions 30 seconds prior to a wind gust event and 30 seconds after a wind gust event. This was achieved by investigating relative lane position to the roadway dividing line, and the mean standard deviation within the lane. This analysis of lane keeping was similar to Kubose et al. (2006), and Gershon et al. (2009) Analysis was also conducted on the recall of billboards presented during the drive.

\section{RESULTS}

Analysis of lane keeping and lane deviation data was done using four separate 3(Task type)x2(30seconds prior, 30 seconds post) Mixed-ANOVAs with task type as the between subjects factor and 30 seconds prior and 30 seconds post wing gust as the within subjects factor.

Mean lane position for time period 1. Results did not indicate any significant or marginally significant main effects or interaction effects for the lane position of the vehicle relative to the roadway dividing line.

Mean lane position for time period 2. Results indicated a significant main effect for task type $F(2,27)=6.15, p<.01, M S E=2.54$; partial- $\eta^{2}=.31$. Participants in the late conversation task 
$(M=6.12 \mathrm{~m})$ were driving closer to the roadway diving line as compared to participants in the full conversation task $(M=7.48 \mathrm{~m})$, and the no conversation task $(M=7.77 \mathrm{~m})$. There was no significant main effect for time, and there were no significant interactions between factors. Simple effect analysis using univariate ANOVA indicated that there were significant differences between task types 30 seconds prior to the wind gust event. $F(1,27)=11.29, p<.001, M S E=$ 1.27; partial- $\eta^{2}=.46$ but no differences between task types 30 seconds after the wind gust event $F(1,27)=1.10, p=.35, M S E=2.96$; partial $-\eta^{2}=.08$ Follow up pairwise comparisons were conducted on the first 30 seconds to investigate differences between the tasks. Results indicated that in the initial 30 seconds, participants in the no conversation task were driving significantly further from the roadway dividing line compared to the participants in late conversation task $F(1,27)=19.60, p<.001$. It was also observed that participants in the full conversation task were also driving further to the right of the roadway dividing line as compared to participants in the late conversation task $F(1,27)=13.73, p<.001$. There was no significant difference between participants in the no conversation task and participants in the full conversation task.

$\underline{\text { Time Period } 1}$

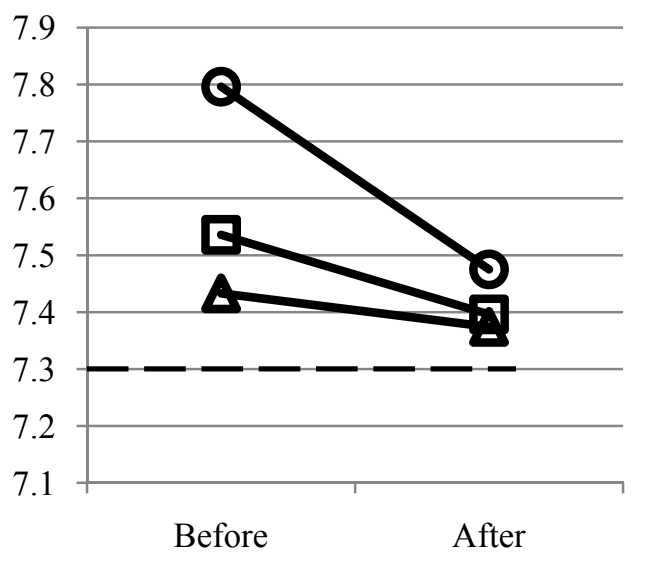

ONo conversation

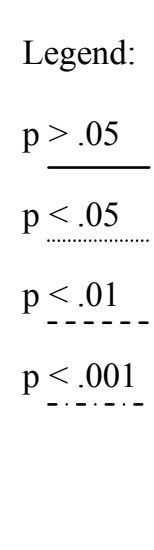

Full Conversation $\underline{\text { Time Period } 2}$

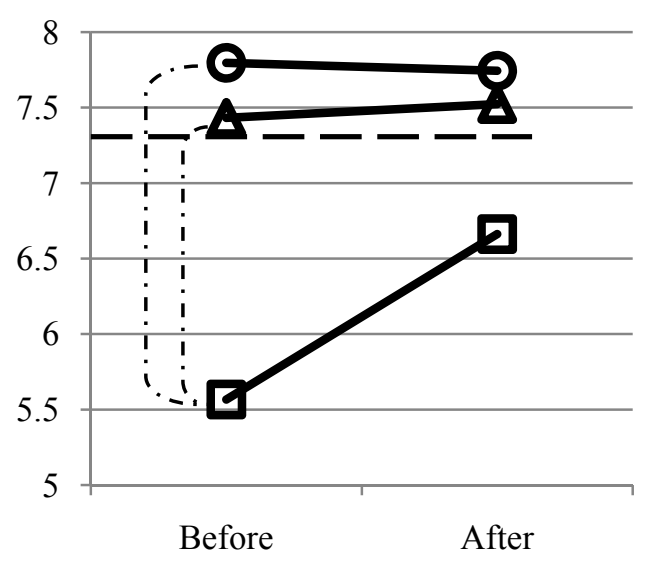

$\triangle$ Late Conversation

Figure 1. Lane position (in meters) before and after wind gust event between groups. Larger numbers on the ordinate indicate increasing distance from roadway dividing line. Dashed line indicates safe lane position i.e. Right lane.

Mean standard deviation lane position for time period 1. Results indicated a marginally significant main effect for time, $F(1,27)=3.93, p=.06, M S E=0.15$; partial- $\eta^{2}=.13$. There was more variability in the 30 seconds prior $(M=0.98)$ to the wind gust event as compared to the 30 seconds post $(M=0.78)$ wind gust event.

Planned comparisons with least significant difference adjustments revealed that lane variability decreased significantly 30 seconds prior to the wind gust as compared to 30 seconds post wind gust for the full conversation task, $F(1,27)=6.42, p<.05$. There were no significant changes in lane variability in the no conversation task and late conversation task.

Mean standard deviation lane position for time period 2. Results indicated a significant main effect for time $F(1,27)=10.89, p<.01, M S E=0.24$; partial $-\eta^{2}=.29$. Lane variability was greater 30 seconds prior to the wind gust $(M=0.87)$ as compared to 30 seconds post wind gust $(M=0.46)$ There was also a marginally significant interaction effect, $F(1,27)=3.12, p=0.06$, $M S E=0.24$; partial- $\eta^{2}=.19$. 
Planned comparisons with least significant difference adjustments revealed that there was a significant decrease in lane variability 30 seconds after the wind gust event as compared to 30 seconds before the wind gust event for the no conversation group $F(1,27)=6.29, p<.05$. This decrease in lane variability was also observed in the full conversation group $F(1,27)=10.84, p$ $<.01$.

Simple effects analysis using univariate ANOVA indicated that there were significant differences between task types 30 seconds prior to the wind gust event. $F(1,27)=4.66, p<.05$, $M S E=0.17$; partial- $\eta^{2}=.26$ but no differences between task types 30 seconds after the wind gust event $F(1,27)=.51, p=.6, M S E=0.23$; partial $-\eta^{2}=.03$ Follow up pairwise comparisons were run on the initial 30 seconds prior to the wind gust. Results indicated that in the initial 30 seconds, drivers in the full conversation task were driving more erratically compared to the participants in late conversation task $F(1,27)=8.99, p<.01$. There was also a marginal difference between the no conversation task and late conversation task $F(1,27)=3.95, p=.06$. There was no significant difference between the no conversation task and full conversation task during this period.

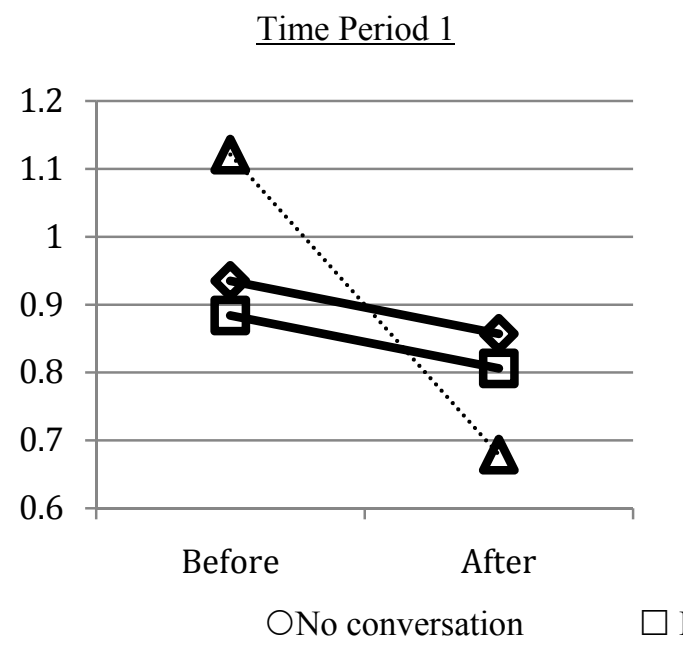

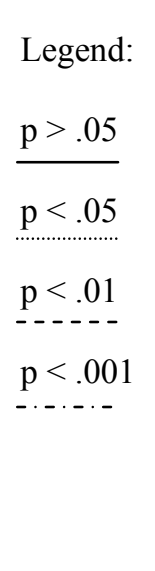

Full Conversation
Time Period 2

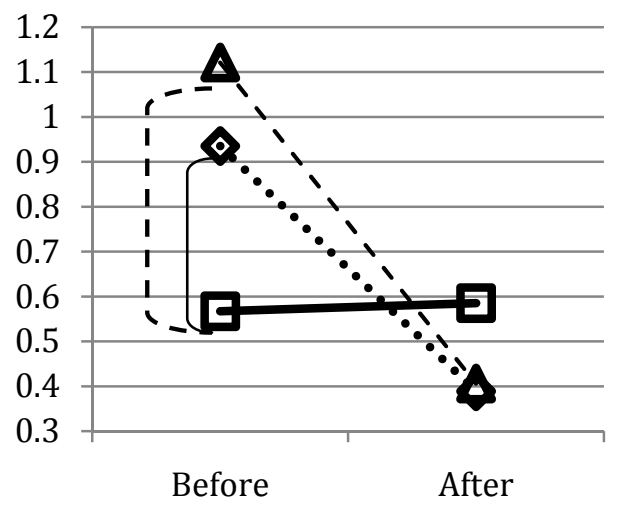

$\triangle$ Late Conversation

Figure 2. Mean lane deviations before and after wind gust event between groups. Larger numbers on the ordinate indicate greater variability.

Memory task. A one-way ANOVA was used to measure potential differences in driver's ability to recall billboards that appeared on the road shoulder during the drive. Results indicated a difference between task types, $F(2,27)=4.71, p<.05$ Follow up pairwise comparisons indicated that the participants in the no conversation task $(M=3, S D=0.82)$, were able to recall more billboard images than participants in the full conversation task $(M=1.7, S D=1.16), F(1,27)=$ $6.52, p<.05$. Results also indicated that participants in the late conversation task $(M=3.1, S D=$ $1.37)$, were able to recall more billboard images than participants in the full conversation task $(M$ $=1.7, S D=1.16), F(1,27)=7.56, p<.05$.

\section{DISCUSSION}

Mean lateral lane position. There were no significant changes in driving behavior during the first time period, before or after the wind gust. This was surprising as no dual task interference effects were observed in the full conversation task during this period, as would be expected from 
participants who engaged in a conversation task. However, during the second time period, participants in the late conversation task appeared to be driving partially in the left lane i.e. closer to the roadway dividing line as compared to the no conversation and full conversation task prior to the wind gust event. This poorer lane position might be indicative of dual task interference from the conversation task. Interestingly, participants in the full conversation task did not show any significant difference when compared to participants in the no conversation task. This might be indicative that given sufficient practice, individuals might be able to successfully time share resources. Though the results might suggest that engaging in a prolonged conversation might improve performance in a monotonous drive, it would be spurious to do so.

Mean standard deviation of lane position. Drivers in the full conversation task showed a significant decrease in lane variability in the first time block after the wind gust, bringing their variability to levels similar to drivers who were not conversing. This might indicate that although their attention might have been divided, they were still able to refocus their attention to the handling of the vehicle. This effect could be likened to a post-error return to attention or in the case of the wind gust, a potentially dangerous event. Overall results seem to suggest that after the wind gust event, participants were less erratic in their driving. This might indicate a possible return of attention to the driving task (Jentzsch, \& Dudschig, 2009). Interestingly, during the second watch period, participants in the late conversation task did not show any significant changes in lane variability, as compared to participants in the other tasks. Firstly, driver variability 30 seconds prior to the wind gust was lesser in the late conversation task as compared to the other tasks. This was interesting in the light of being engaged in a conversation, as it was expected that participants would be more erratic in their driving as observed in participants in the first watch period of the full conversation task. This consistent lane keeping behavior was also noted 30 seconds after the wind gust event, suggesting that the wind gust had no effect on participants in the late conversation task. A possible explanation for driver stability could be attributed to the potential for a secondary cognitive task to improve postural stability (Swan, Otani, Loubert, Sheffert, \& Dunbar, 2004). The energizing or alerting effect of the wind gust was also clearly visible in the no conversation and full conversation task as their lane keeping variability decreased significantly 30 seconds after the wind gust.

Recall task. Participants in the dual task conditions were unable to recognize as many signs when compared to a single task condition. Results from the current study indicate the same results. This finding was further extended whereby participants in the late conversation condition had seen all the signs prior to the conversation task, indicating that prior to the conversation, participants were able to encode the signs seen during the drive in the same fashion as participants not engaged in conversation. The current study also lends further support to data found by Strayer, Drews and Johnston (2003).

\section{CONCLUSION}

Results with respect to lane keeping and lane deviation remain inconclusive. The results do suggest that participants when faced with sudden shifts in road conditions e.g. wind gusts, were able to return their attention to the primary task of driving, regardless of whether they were engaged in a conversation or not. This might also suggest that cell phone conversations while driving might not be as detrimental as expected. Given that this finding might be indicative of a possible positive effect of cell phone conversation, though the results of the memory task suggest 
otherwise. Although there might some benefit to the driving task in general, the lack of an ability to recall common billboard on the roadway suggest a possible decreased visual attention to the roadway. This result was supported by findings by Atchley and Dressel (2004). With the current state of knowledge, it would remain wise to not engage in a cell phone conversation while driving regardless of road conditions as the perceived benefits still do not outweigh its costs.

\section{REFERENCES}

Atchley, P., \& Dressel, J. (2004). Conversation limits the functional field of view. Human Factors, 46(4), 664-673.

Bradley, M.M., \& Lang, P.J. (1999). Affective norms for English words (ANEW). Gainesville, FL. The NIMH Center for the Study of Emotion and Attention, University of Florida.

Chan, M. (2008). Benefits and cost of dual-tasking in a vigilance task: A laboratory and driving simulator investigation. Unpublished manuscript. University of Kansas, Lawrence, KS.

Davies, D. R., \& Parasuraman, R. (1982). The Psychology of Vigilance. London: Academic Press Inc.

Drory, A. (1985). Effects of rest and secondary task on simulated truck-driving task performance. Human Factors, 27(2), 201-207.

Gershon, P., Ronen, A., Oron-Gilad, T., \& Shinar, D. (2009). The effects of an interactive cognitive task (ICT) in suppressing fatigue symptoms in driving. Transportation Research Part F, 12, 21-28.

Jentzsch, I., \& Dudschig, C. (2009). Why do we slow down after an error? Mechanisms underlying the effects of posterror slowing The Quarterly Journal of Experimental Psychology, 62(2), 209-218.

Kubose, T. T., Bock, K., Dell, G.S., Garnsey, S.M., Kramer, A.F., \& Mayhugh, J. (2006). The effects of speech production and speech comprehension on simulated driving performance. Applied Cognitive Psychology, 20, 43-63.

Strayer, D. L., Drews, F.A., \& Johnston, W.A. (2003). Cell phone-induced failures of visual attention during simulated driving. Journal of Experimental Psychology: Applied, 9(1), 2332 .

Strayer, D. L., \& Johnston,W.A. (2001). Driver to distraction: Dual-task studies of simulated driving and conversing on a cellular telephone. Psychological Science, 12(6), 462-466.

Swan, L., Otani, H., Loubert, P.V., Sheffert, S.M., \& Dunbar, G.L. (2004). Improving balance by performing a secondary cognitive task. British Journal of Psychology, 95, 31-40.

Thiffault, P., \& Bergeron, J. (2003). Monotony of road environment and driver fatigue: a simulator study. Accident Analysis and Prevention, 35, 381-391.

Yerkes, R. M., \& Dodson, J.D. (1908). The relation of strength of stimulus to rapidity of habitformation. Journal of Comparative Neurology and Psychology, 18, 459-482. 